\title{
Topographic Diagnosis of Craniopharyngiomas: The Accuracy of MRI Findings Observed on Conventional T1 and T2 Images
}

\author{
(D) R. Prieto, (D).M. Pascual, and (D) B. Barrios
}

\begin{abstract}
BACKGROUND AND PURPOSE: The topography of craniopharyngiomas has proved fundamental in predicting the involvement of vital brain structures and the possibility of achieving a safe radical resection. Beyond the imprecise term "suprasellar," indiscriminately used for craniopharyngiomas, an accurate definition of craniopharyngioma topography should be assessed by preoperative MR imaging. The objective of this study was to investigate the MRI findings that help define craniopharyngioma topography.
\end{abstract}

MATERIALS AND METHODS: This study retrospectively investigated a cohort of 200 surgically treated craniopharyngiomas with their corresponding preoperative midsagittal and coronal conventional T1- and T2-weighted MR images, along with detailed descriptions of the surgical findings. Radiologic variables related to the occupation of the tumor of intracranial compartments and the distortions of anatomic structures along the sella turcica-third ventricle axis were analyzed and correlated with the definitive craniopharyngioma topography observed during the surgical procedures. A predictive model for craniopharyngioma topography was generated by multivariate analysis.

RESULTS: Five major craniopharyngioma topographies can be defined according to the degree of hypothalamic distortion caused by the tumor: sellar-suprasellar, pseudointraventricular, secondary intraventricular, not strictly intraventricular, and strictly intraventricular. Seven key radiologic variables identified on preoperative MRI allowed a correct overall prediction of craniopharyngioma topography in $86 \%$ of cases: 1) third ventricle occupation, 2) pituitary stalk distortion, 3) relative level of the hypothalamus in relation to the tumor, 4) chiasmatic cistern occupation, 5) mammillary body angle, 6) type of chiasm distortion, and 7) tumor shape.

CONCLUSIONS: Systematic assessment of these 7 variables on conventional preoperative $\mathrm{T} 1$ and T2 MRI is a useful and reliable method to ascertain individual craniopharyngioma topography.

ABBREVIATIONS: $\mathrm{CP}$ = craniopharyngioma; $\mathrm{CRT}=$ Classification and Regression Tree; $\mathrm{LR}=$ likelihood ratio; $\mathrm{MBA}=$ mammillary body angle; $\mathrm{PS}=$ pituitary stalk; $3 V=$ third ventricle; $T V F=$ third ventricle floor

$C^{r}$ aniopharyngiomas (CPs) remain a neurosurgical challenge. This type of tumor is characterized by extreme heterogeneity regarding clinical expression, pathologic features, and topography. The vague, general description of CPs as "suprasellar" tumors has frequently led to imprecise topographic diagnoses. ${ }^{1,2}$ The use of the term "suprasellar" dates to the early 20th Century, when the presence of calcifications above the sella turcica on skull radiographs was the fundamental radiologic sign indicating $\mathrm{CP}$

Received May 16, 2017; accepted June 30.

From the Department of Neurosurgery (R.P.), Puerta de Hierro University Hospital, Madrid, Spain; Department of Neurosurgery (J.M.P.), La Princesa University Hospital, Madrid Spain; and Statistics Department (L.B.), Computing Center, Consejo Superior de Investigaciones Científicas, Madrid, Spain.

Please address correspondence to Ruth Prieto, MD, PhD, Department of Neurosurgery, Puerta de Hierro University Hospital, C/Joaquín Rodrigo 2, Majadahonda, 28222, Madrid, Spain; e-mail: rprieto29@hotmail.com

三 Indicates article with supplemental on-line tables and appendix.

http://dx.doi.org/10.3174/ajnr.A5361 diagnosis. Currently, the incorrect assumption of a primary suprasellar position for most CPs might lead to improper surgical planning. Therefore, an accurate topographic classification of individual cases should be established preoperatively. ${ }^{2}$

The potential for CP development at any point along the vertical hypothalamic-hypophyseal axis, from the sella turcica to the third ventricle $(3 \mathrm{~V})$, determines the wide range of topographies displayed. Certainly, current MR imaging technology provides a tool allowing an accurate definition of CP topography in most cases. $^{2,3}$ The specific anatomic relationships between CPs and the $3 \mathrm{~V}$ floor and walls, where the vital hypothalamic nuclei are located, are an essential factor for assessing the possibility of achieving a safe radical removal. ${ }^{1-5}$ Consequently, defining the topographic relationships between the tumor and the third ventricle floor (TVF) should be one major objective of preoperative MR imaging in patients with CP.

In 2004, our group developed a topographic classification system based on the types of TVF distortion caused by CPs. ${ }^{1-4}$ This 
scheme has proved helpful to preoperatively ascertain the anatomic position of the hypothalamus and its degree of distortion, information that is correlated with surgical risks and patient outcome. In previous studies, we have been able to identify some specific MR imaging signs invaluable for defining the true CP-3V relationships, such as the mammillary body angle (MBA) and the type of optic chiasm distortion. ${ }^{6,7}$ The aim of the present study was to analyze the diagnostic power of conventional T1- and T2weighted sequences for an accurate depiction of CP topography on midsagittal and transinfundibular-coronal MR images. Specifically, we have examined the preoperative MR imaging findings that best predict the type of $\mathrm{CP}-3 \mathrm{~V}$ relationships present in each patient.

\section{MATERIALS AND METHODS \\ Case Series}

The cases included in this study were selected from a recent publication in which we analyzed CP topography in a series of 500 cases and correlated it with the patterns of tumor adhesion to the hypothalamus. ${ }^{5}$ A total of $200 \mathrm{CPs}$ published in the MR imaging era (1990-2016) were selected from this cohort, all meeting the following inclusion criteria: 1) CP diagnosis was confirmed pathologically, 2) preoperative midsagittal and transinfundibular-coronal MR images were displayed in the reports, and 3) the patient was surgically treated, and a detailed description and photographic evidence of the tumor relationships with the TVF/hypothalamus was provided (On-line Table 1; On-line Appendix).

\section{Topographic Classification of CPs}

The tumors were classified into 5 major categories, according to our classification scheme of CP-3V relationships ${ }^{1-5}: 1$ ) sellar-suprasellar CPs, which include tumors occupying exclusively the sellar and/or suprasellar compartments beneath an intact TVF;2) suprasellar-pseudointraventricular CPs, suprasellar lesions causing an upward displacement of the TVF, mimicking an intraventricular position; 3) secondary intraventricular CPs, lesions developing initially beneath the $3 \mathrm{~V}$ but invading the $3 \mathrm{~V}$ after breaking through the TVF; 4) infundibulo-tuberal or not strictly intraventricular CPs, tumors developed originally within the nervous tissue of the TVF itself (within the infundibulum and/or tuber cinereum), which predominantly expand within the $3 \mathrm{~V}$ at later stages; and 5) strictly intraventricular CPs, tumors primarily originating within the $3 \mathrm{~V}$, above an intact TVF (Fig 1). The final CP topographic categorization for the 200 cases constituting our cohort was established in a previous article that analyzed the correlation of intraoperative anatomic and pathologic findings with pre- and postoperative MR imaging studies. ${ }^{5}$ In the present study, we performed a "blinded" analysis of the MR imaging variables used to ascertain the CP topography-that is, in each case, we evaluated the different MR imaging variables without knowing the definitive topography confirmed in our previous article.

\section{Surgical Treatment and Outcome}

Surgical routes chosen for each case were recorded and postoperative outcome was classified into 4 categories: 1) good, long-term survival without new neurologic deficits; 2) fair, new but not disabling deficits, including panhypopituitarism or diabetes insipidus; 3) poor, new neurologic symptoms that considerably impair the quality of life; and 4) death within the first month after surgery.

\section{Selection and Categorization of Variables Analyzed on Preoperative MR Images}

The size, shape, and consistency of the tumor, in addition to the occupation of intracranial compartments and distortion of anatomic structures along the sella-3V axis, were evaluated on midsagittal and coronal conventional T1- and T2-weighted MR images. Occupation of the sella turcica and chiasmatic cistern was classified into 3 categories (tumor-free, partially occupied, and wholly occupied), whereas only 2 groups were considered regarding the occupation of the $3 \mathrm{~V}$ (tumor-free or occupied). The appearance of the pituitary stalk (PS) was categorized as follows: 1) wholly visible, intact PS; 2) infiltrated by tumor, thickened PS due to macroscopic tumor infiltration; 3) amputated, PS upper portion not visible due to tumor growth; and 4) not visible, unrecognizable PS due to tumor encroachment. The relative anatomic position between the hypothalamus and the $\mathrm{CP}$, best assessed on coronal MR imaging sections, was classified into 3 groups: 1) below the lower tumor pole level, 2) around its midthird portion, and 3) above the upper third of the tumor. We measured the mammillary body angle, formed by the intersection of a plane tangential to the base of the mammillary bodies with the plane tangential to the fourth ventricle floor, on the midsagittal MR images. $^{6}$

Finally, the chiasm distortion caused by the tumor was evaluated and classified according to the scheme published by our group in 2013: 1) not distorted, normal position and shape; 2) compressed downward, flattened chiasm at the anteroinferior margin of the tumor; 3) compressed forward, flattened chiasm between the tumor and the tuberculum sellae; 4) stretched forward, elongated along the anterior margin of the tumor; 5) stretched upward, elongated chiasm along the superior surface of the tumor; 6) stretched backward, elongated along the posterior margin of the tumor; and 7) not visible, completely unrecognizable.

\section{Statistical Analysis}

Bilateral correlations between pairs of categoric variables were assessed with the asymptotic $\chi^{2}$, the Monte Carlo exact, or the linear-by-linear association tests. A multivariate Classification and Regression Tree (CRT) growing method was then applied to explain CP topography. This hierarchic stepwise procedure creates a decision tree by splitting the cohort into 2 branches at each step to maximize the homogeneity of case subgroups within each branch. At each step, the CRT chooses the nominal variable showing the strongest independent interaction with the topographic categories included in the previous step. Then, we used a multiple correspondence analysis to investigate the power of the radiologic variables selected in the first and second steps by the CRT method. Finally, we quantified the accuracy of the multivariate MR imaging model generated to discriminate among the $5 \mathrm{CP}$ categories. For that purpose, we used the likelihood ratio (LR) for positive test results $[\mathrm{LR}+=$ sensitivity/(1-specificity) $]$ and $[\mathrm{LR}-=(1-$ sensitivity)/specificity] for negative test results. Statistical analyses 


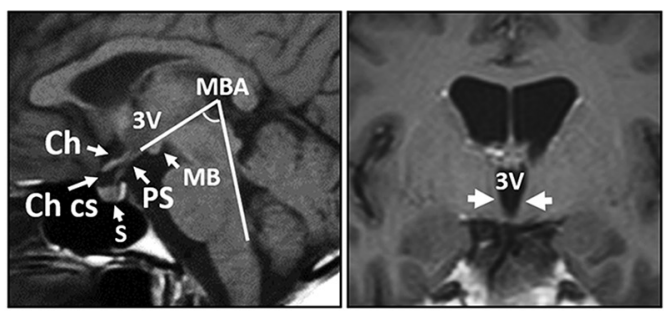

Sellar-Suprasellar
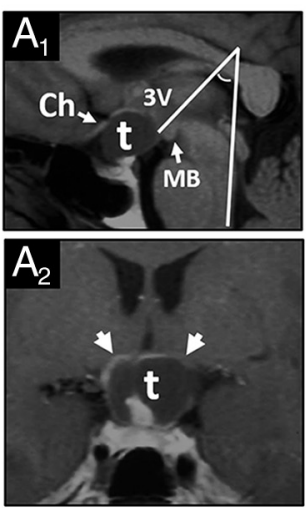

\begin{tabular}{l|c} 
& Sellar-Suprasellar \\
\hline 3V & Free \\
Ch cistern & Wholly occupied \\
Stalk & Not visible \\
Hypothal. & Upper-third \\
MBA & $60-89^{\circ}$ \\
Chiasm & Stretched upward \\
CP shape & Round, Pear-like, Dumbbell
\end{tabular}

Pseudo - 3V
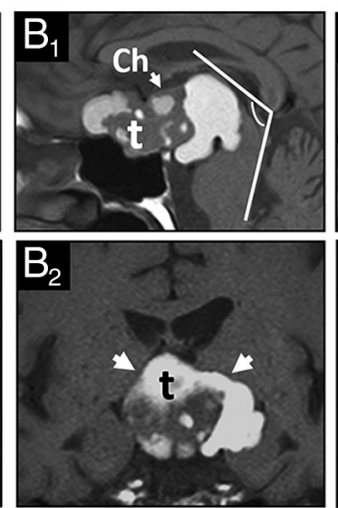

Pseudo-3V

Occupied

Wholly occupied

Not visible

Upper-third $\geq 90^{\circ}$

Stretched upward

Multilobulated, Pear-like
Secondary - 3V
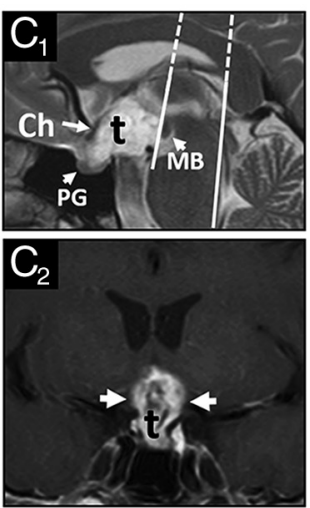

Secondary-3V

Occupied

Not visible

Middle-third 30-59

Stretched forward

Multilobulated, Elliptical
3V - Not Strict
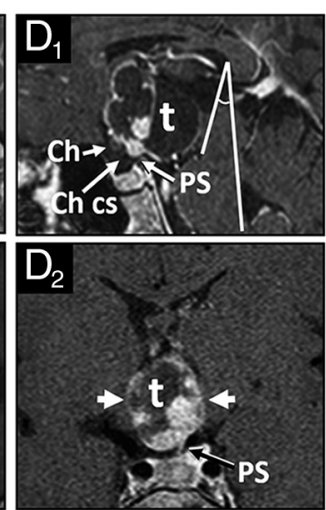

3V-Not Strict

Occupied

Partially occupied

Amputated, Infiltrated

Middle-third

$<30^{\circ}$

Compressed forward

Elliptical, Round
3V - Strict
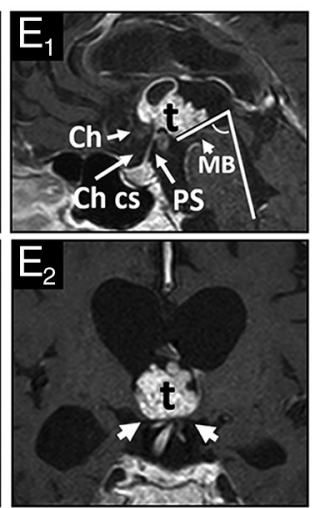

3V-Strict

Occupied

Free

Wholly visible

Lower-third

30-59

Compressed downward

Round

FIG 1. Preoperative MR imaging assessment to accurately define the 5 major CP topographies. The upper images correspond to midsagittal and transinfundibular-coronal T1-weighted MR images from a healthy adult. Ch indicates chiasm; Ch cs, chiasm cistern; MB, mammillary body. The middle (1, sagittal) and lower (2, coronal) rows show MR images of the 5 CP topographic categories considered. A, Sellar-suprasellar CP: the Ch cistern is occupied by a round tumor, the PS is not visible, the chiasm is stretched upward, and the hypothalamus (arrows) is above the tumor. $B$, Pseudointraventricular CP: the sella, the Ch cistern, and the $3 \mathrm{~V}$ are occupied by a multilobulated tumor. The PS is not visible, the MBA is obtuse, and the hypothalamus (arrows) is over the upper third of the tumor. C, Secondary intraventricular CP: the Ch cistern and the 3V are occupied by the tumor, the PS is not visible, the chiasm is stretched forward, and the hypothalamus (arrows) is around the midthird of the tumor. $D$, Infundibulo-tuberal or not strictly intraventricular CP: the Ch cistern is partially occupied by tumor, the PS is amputated, the chiasm is compressed forward, the MBA is hyperacute, and the hypothalamus (arrows) is around the midthird of the tumor. E, Strictly intraventricular CP: the Ch cistern is tumor-free, the PS is entirely visible, the chiasm is compressed downward, and the hypothalamus (arrows) is below the lower third of the tumor. The Table summarizes the most typical MR imaging characteristics for each topographic category. T indicates tumor.

were performed using SPSS software (Version 23; IBM, Armonk, New York).

\section{RESULTS}

\section{Topographic Distribution and Surgical Outcome}

On-line Table 2 shows the distribution of the topographic categories included in this cohort of 200 CPs. The infundibulo-tuberal or not strictly intraventricular category was the predominant one $(32.5 \%, n=65)$, followed by the strictly intraventricular $(21 \%, n=42)$. The remaining lesions originating from the pituitary gland-stalk complex beneath the TVF were categorized into the sellar-suprasellar $(n=37)$, pseudointraventricular $(n=32)$, and secondary intraventricular $(n=24)$ topographies.

All patients underwent surgical treatment. The most frequent surgical routes used were basal pterional/subfrontal (31\%) and transsphenoidal (33\%). Postoperative outcomes were available in 155 of 200 patients. Most cases had a satisfactory long-term outcome: good in $41 \%$ and fair, with minor disturbances, in $43 \%$ of cases. Last, an overall 3\% mortality $(n=5)$ was registered within the first month following the surgical procedure. Poor outcomes and deaths reported in our cohort were related to the hypothalamic injury inflicted by surgical maneuvers in all cases, a finding analogous to those reported in large surgical series published in the MR imaging era. ${ }^{8}$

\section{Variables Analyzed on Conventional Midsagittal and Coronal MR Imaging}

On-line Table 2 presents the distribution of the major radiologic features analyzed on preoperative T1- and T2-weighted MR images. The most common $\mathrm{CP}$ shape in this cohort was round (42\%), followed by elliptical $(22.5 \%)$ and multilobulated $(22.5 \%)$. The sella turcica was tumor-free in almost $60 \%$ of the cases and wholly occupied in $25.5 \%$. The chiasmatic cistern was tumor-free in $33 \%$ of the cases, showed a partial occupation in $23.5 \%$, and was totally filled in $43.5 \%$. In this cohort, the $3 \mathrm{~V}$ was occupied by the tumor in almost $90 \%$ of cases.

AJNR Am J Neuroradiol 38:2073-80 Nov 2017 www.ajnr.org 
Bivariate relationships between the variables analyzed on preoperative MRI and CP topography

\begin{tabular}{|c|c|c|}
\hline MRI Variables ${ }^{\mathrm{a}}$ & $P$ Value & Specific Relationships \\
\hline \multirow[t]{2}{*}{ 3V occupation } & $<.001$ & Free: highest rate in Sellar-SS (57\%) \\
\hline & & Occupied: in $100 \%$ of Pseudo-3V, Secondary, 3V-Not-Strict, and 3V-Strict cases \\
\hline \multirow[t]{4}{*}{ Stalk visualization } & $<.001$ & Visible (wholly identifiable): highest rate in 3V-Strict (79\%) \\
\hline & & Infiltrated: highest rate in 3V-Not-Strict (23\%) \\
\hline & & Amputated (lower half visible): highest rate in 3V-Not-Strict (68\%) \\
\hline & & Not visible: highest rates in Sellar-SS (92\%\%), Pseudo-3V (100\%), and Secondary-3V (92\%) \\
\hline \multirow{3}{*}{$\begin{array}{l}\text { Hypothalamus level relative } \\
\text { to the CP }\end{array}$} & $<.001$ & Lower third: highest rate in $3 \mathrm{~V}$-Strict $(82 \%)$ \\
\hline & & Middle: highest rates in 3V-Not-Strict (91\%) and Secondary-3V (77\%) \\
\hline & & Upper third: highest rates in Sellar-SS (100\%) and Pseudo-3V (91\%) \\
\hline \multirow[t]{3}{*}{ Chiasmatic cistern } & $<.001$ & Free: highest rate in $3 \mathrm{~V}$-Strict $(98 \%)$ \\
\hline & & Partially occupied: highest rate in 3V-Not-Strict (54\%) \\
\hline & & Wholly occupied: highest rates in Sellar-SS (86.5\%), Pseudo-3V (97\%), and Secondary-3V (75\%) \\
\hline \multirow[t]{4}{*}{ MBA } & $<.001$ & $<30^{\circ}$ : highest rate in $3 \mathrm{~V}-$ Not-Strict $(43 \%)$ \\
\hline & & $30^{\circ}-59^{\circ}$ : highest rates in Secondary-3V (58\%) and 3V-Strict (55\%) \\
\hline & & $60^{\circ}-89^{\circ}$ : highest rate in Sellar-SS (64\%) \\
\hline & & $\geq 90^{\circ}$ : highest rate in Pseudo-3V (89\%) \\
\hline \multirow[t]{4}{*}{ Chiasm distortion } & $<.001$ & Compressed downward: highest rates in 3V-Strict (66\%) and 3V-Not-Strict (33\%) \\
\hline & & Compressed forward: highest rate in 3V-Not-Strict (40\%) \\
\hline & & Stretched forward: highest rate in Secondary-3V (50\%) \\
\hline & & Stretched upward: highest rates in Sellar-SS (81\%) and Pseudo-3V (70\%) \\
\hline \multirow[t]{5}{*}{ Tumor shape } & $<.001$ & Round: highest rate in $3 \mathrm{~V}$-Strict $(76 \%)$ \\
\hline & & Elliptical: highest rate in 3V-Not-Strict (37\%) \\
\hline & & Multilobulated: highest rates in Pseudo-3V (62.5\%) and Secondary-3V (62.5\%) \\
\hline & & Pearlike: highest rates in Sellar-SS (30\%) and Pseudo-3V (19\%) \\
\hline & & Dumbbell: highest rates in Sellar-SS (13.5\%) \\
\hline
\end{tabular}

Note:-SS indicates suprasellar; 3V-Not-Strict, not strictly intraventricular or infundibulo-tuberal; Pseudo-3V, suprasellar pseudointraventricular; Secondary-3V, secondary intraventricular category; $3 \mathrm{~V}$-Strict, strictly intraventricular.

a Only the 7 variables included in the multivariate predictive model are shown.

The PS could not be identified in almost half of the cases (46.5\%), owing to the occupation of the chiasmatic cistern by tumoral tissue. It had a normal appearance in only $18.5 \%$ of studies, whereas in $27 \%$, its upper portion was not visible due to tumor growth (a sign we term "PS amputation"). In 8\%, the PS appeared thickened due to macroscopic infiltration by the tumor. The position of the hypothalamus relative to the $\mathrm{CP}$ was observed around the lower pole of the $\mathrm{CP}$ in $19 \%$, around the equator or middle third of the tumor in $42 \%$, and above its upper third in $39 \%$ of cases. The MBA could be measured in $84.5 \%$ of the cases. This angle was hyperacute $\left(<30^{\circ}\right)$ in $18 \%$ of cases, moderately acute $\left(30^{\circ}-59^{\circ}\right)$ in $31.5 \%$, slightly acute $\left(60^{\circ}-89^{\circ}\right)$ in $19.5 \%$, and obtuse $\left(\geq 90^{\circ}\right)$ in $15.5 \%$. Finally, the optic chiasm could be identified in $88.5 \%$ of midsagittal MR images and was grossly deformed in most cases. The 2 most common types of chiasm distortion were an upward stretching by CPs originating beneath the TVF (26\%) and a downward compression by intraventricular CPs (24\%).

\section{Correlation between CP Topography and Surgical Outcome}

A significant correlation between CP topography and surgical outcome was found in this cohort $(P<.034)$, a fact related to the different degree of hypothalamic involvement associated with each topographic category. ${ }^{5}$ The most favorable outcomes were reported for the sellar-suprasellar and pseudointraventricular topographies (good or fair outcomes in $>90 \%$ ), both characterized by a meningeal layer interposed between the tumor and the TVF. Conversely, the worst outcomes were found among patients with CPs showing a secondary intraventricular location (poor outcome or death, $\approx 40 \%$ ), characterized by their extensive attachments to the hypothalamus. CPs with an intraventricular topog- raphy had moderate outcomes, more favorable for those tumors with a strictly intraventricular location (good outcome, 49\%; poor, $11 \%$ ) than for the not strictly intraventricular cases (good outcome $28 \%$; poor $15 \%$ ) due to the intimate relation of the latter category with the hypothalamic region.

\section{Preoperative Definition of CP Topography: An MR Imaging Multivariate Model}

The specific relationships between CP topography and the most important radiologic features analyzed on preoperative conventional MR images are shown in the Table. At the outset of this study, it was found that all CPs lacking occupation of the $3 \mathrm{~V}(n=$ 21) corresponded to the sellar-suprasellar topography. Consequently, these cases were excluded from further analysis. The remaining 179 cases were included in a hierarchic multivariate analysis using the CRT growing method to explore the optimal combination of MR imaging variables providing the best discrimination model for the 5 topographic categories considered. PS appearance was identified as the variable showing the strongest relation to $\mathrm{CP}$ topography, followed by tumor occupation of the chiasmatic cistern and the level of the hypothalamus relative to the tumor. In further steps, the MBA, the type of chiasm distortion, and tumor shape were selected as additional independent variables to build a predictive model of $\mathrm{CP}$ topography (Fig 2). The predictive power of this model was $83.8 \%$ - that is, a correct overall preoperative classification can be achieved in almost $84 \%$ of cases by accurately defining these 6 radiologic variables on MR images.

After that, a multiple correspondence analysis including the whole cohort of 200 cases was performed to investigate the predictive accuracy of $\mathrm{CP}$ topography achieved with the 3 independent variables selected in the first and second steps by the CRT 


\section{CP Topography}

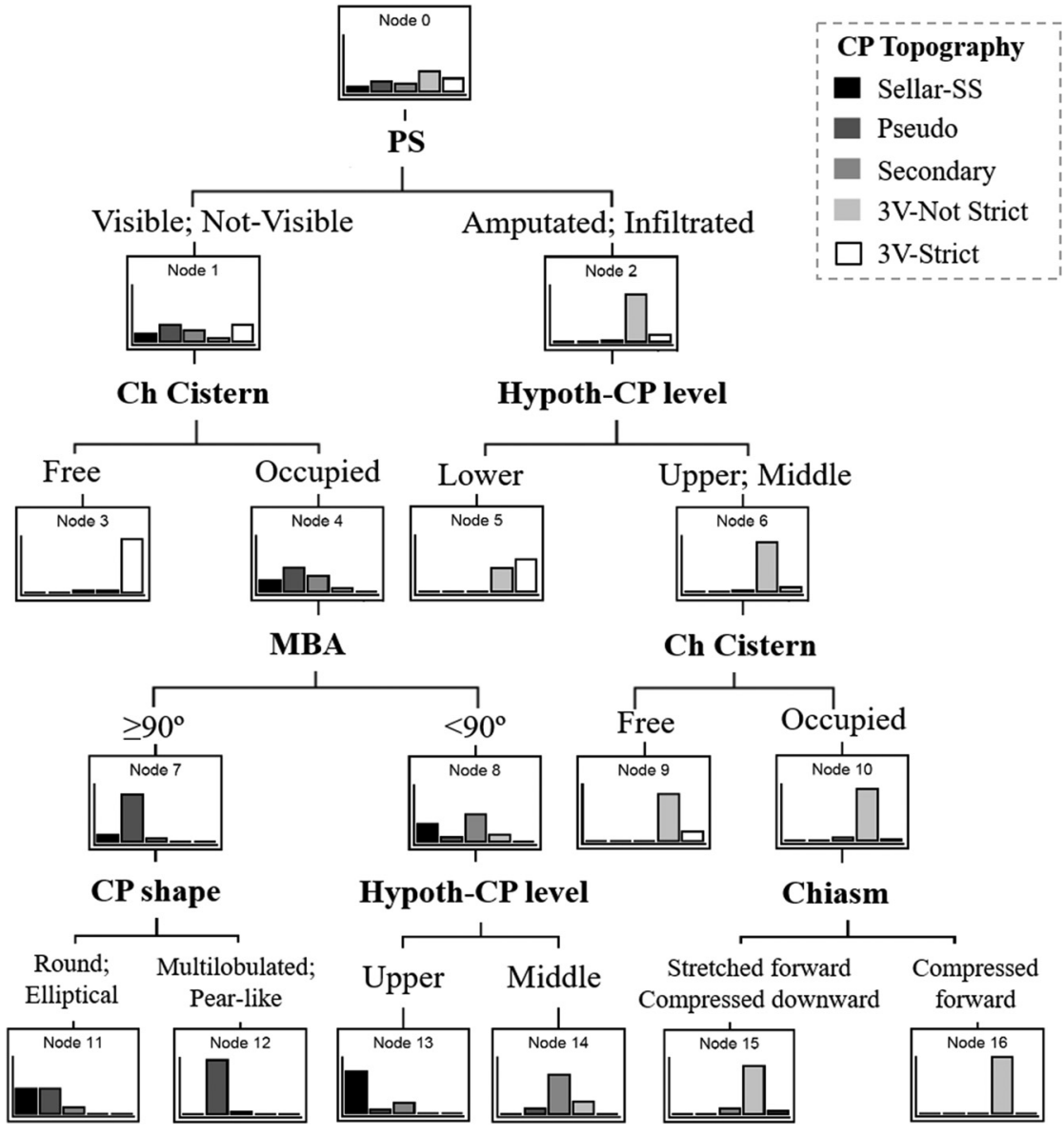

FIG 2. MR imaging predictors of CP topography. This growing decision tree shows the variables selected in a multivariate model to define the CP topography. The appearance of the pituitary stalk on preoperative MR imaging was chosen in the first step. The occupation of the chiasmatic cistern (Ch Cistern), the position of the hypothalamus regarding the tumor (Hypoth-CP level), the MBA, the tumor shape (CP shape), and the chiasm distortion (Chiasm) were selected in the following steps. Note that the probability for the not strictly intraventricular category (3V-Not Strict) significantly increased when the PS was either amputated or infiltrated, the Hypoth-CP level was not in the lower third of the tumor, the Ch cistern was occupied by tumor, and the Chiasm was compressed forward (nodes 2, 6, 10, 16). In contrast, the probability of the strictly intraventricular category (3V-Strict) significantly increased when the Ch cistern was free of tumor (node 3). The probability of the pseudointraventricular topography (Pseudo) significantly increased when the PS cistern was not visible, the Ch cistern was occupied by tumor, the MBA was obtuse $\left(>90^{\circ}\right)$, and the tumor had a multilobulated or pearlike shape (nodes 1, 4, 7, 12). Among the cases with an MBA of $<90^{\circ}$ (node 8), the position of the hypothalamus above the upper third of the tumor indicated the sellarsuprasellar category (Sellar-SS), whereas its position around the middle portion of the tumor suggested a secondary intraventricular topography (Secondary, nodes 13, 14). Correct prediction of CP topography with this multivariate model, including 6 categoric variables studied on preoperative MR imaging, reached $83.8 \%$.

method (PS appearance, hypothalamus level, and chiasmatic cistern occupation). We found that a correct preoperative topographic prediction was achieved in $76.3 \%$ of cases simply by considering these $3 \mathrm{MR}$ imaging variables (Fig 3).

Finally, we assessed the diagnostic accuracy of a multivariate model that included the $7 \mathrm{MR}$ imaging variables to define $\mathrm{CP}$ topography (tumor occupation of the $3 \mathrm{~V}$ in addition to the 6 variables selected by the CRT method). Overall, the percentage of correct classification improved to $86 \%$. The accuracy of this model for discriminating among the 5 topographic $\mathrm{CP}$ categories was quantified with positive $(\mathrm{LR}+)$ and negative $(\mathrm{LR}-)$ likeli- hood ratios. The best discrimination power, characterized by a high LR+ along with a low LR -, was achieved for the sellar-suprasellar, pseudointraventricular, and strictly intraventricular categories. On the contrary, the worst identified category (lowest LR+ and highest LR - values) corresponded to the secondary intraventricular topography (Fig 4).

\section{DISCUSSION}

Craniopharyngioma Topography:

The Importance of an Accurate Preoperative Definition

Neuroradiologists should recognize the extremely heterogeneous pathologic spectrum of CPs, particularly the huge influence that tumor topography pattern has on surgical outcome. The imprecise, generalized description of CPs as "suprasellar lesions" must be replaced by a comprehensive topographic characterization, one capable of predicting the surgical risks associated with each topographic category. ${ }^{2}$ A detailed preoperative understanding of the anatomic relationships between a $\mathrm{CP}$ and the surrounding neurovascular structures is essential to maximize the likelihood of a radical excision while minimizing the risks of undue hypothalamic injury, which is the most devastating complication during CP surgery. . $^{5,8,9}$

This study analyzes the MR imaging findings observed on conventional $\mathrm{T} 1$ and T2 images displayed in the CP reports included in our cohort. In most cases, only midsagittal and coronal scans through the infundibular region of the $3 \mathrm{~V}$ (transinfundibular-coronal) were shown. The lack of a complete set of images obtained in each case can be judged as an obvious limitation of our analysis. However, in our topographic scheme, the fundamental criterion for categorizing CPs is the anatomic relationship between the tumor and the hypothalamus, the critical structure to be preserved in an operation. ${ }^{1-6} \mathrm{CPs}$ are predominately midline lesions developed from epithelial remnants of the Ratkhe pouch placed at any point of the pituitary-hypothalamic axis, usually within the pars tuberalis (a tongue of adenohypophysial tissue enveloping the PS and infundibulum). ${ }^{4}$ The relative original position of these cells with regard to the meningeal layers covering the PS and infundibulum will determine the final CP topography. ${ }^{1,2}$ Consequently, the midsagittal and transinfundibular-coronal MR images represent the 2 basic sections to accurately elucidate the CP topography.

AJNR Am J Neuroradiol 38:2073-80 Nov 2017 www.ajnr.org 


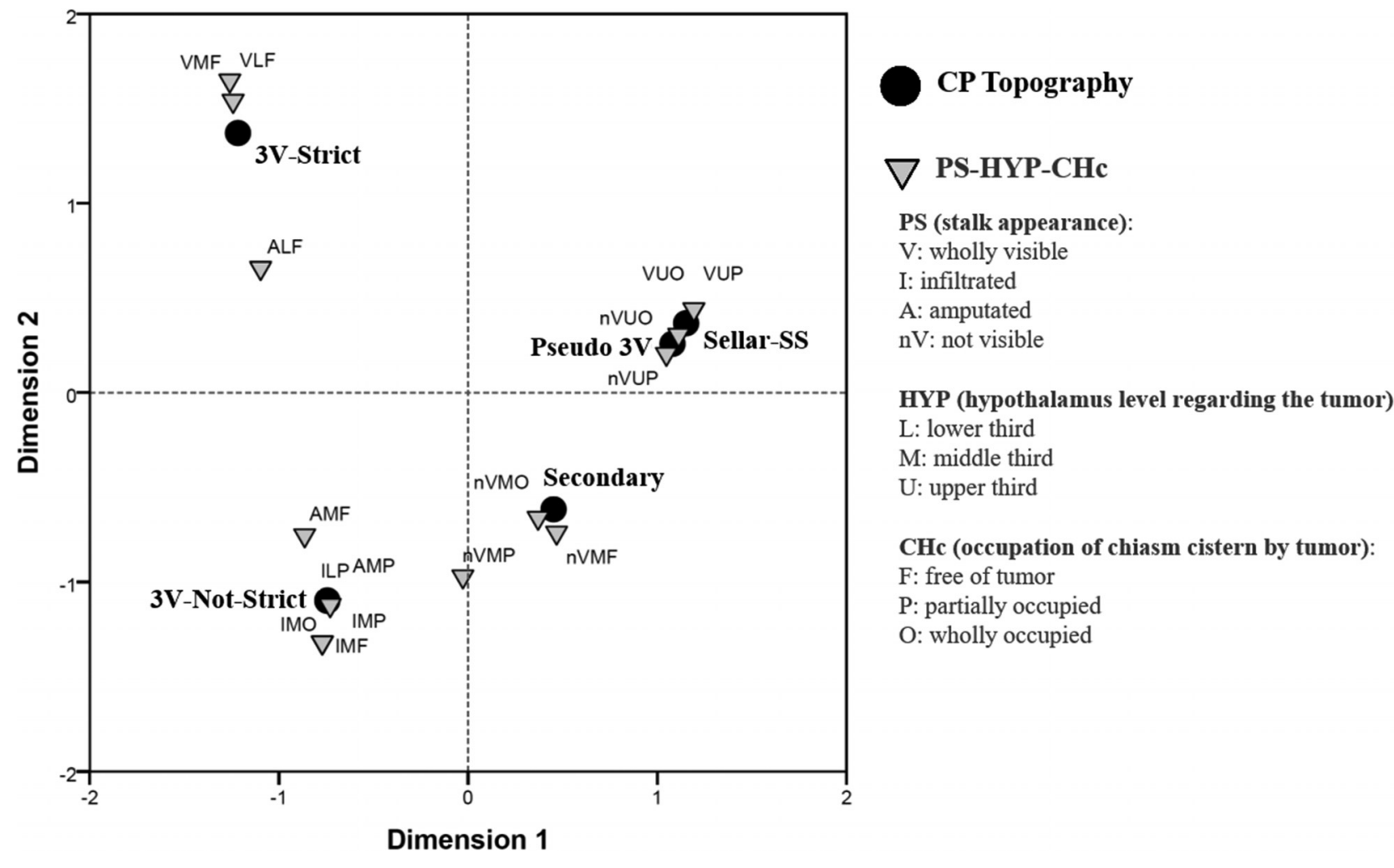

FIG 3. Multivariate model of CP topography for CPs: A 2D plot showing the multiple correspondence analysis between CP topographic categories (black circles) and the appearance of the pituitary stalk, the hypothalamus level in relation to the tumor (HYP), and the occupation of the chiasmatic cistern ( $\mathrm{CHc}$ ) (gray triangles). Dimension 1 separates the intraventricular topographies (strictly and not strictly) from the remaining variables, whereas dimension 2 mostly separates the strictly intraventricular CPs (3V-Strict) from the not strictly ones (3V-Not-Strict). Note that the position of the secondary intraventricular category (Secondary), the most difficult to define preoperatively, is in the trajectory between the 3V-Not-Strict and the sellar-suprasellar (SS) or pseudointraventricular (Pseudo-3V) categories, owing to the radiologic signs that these lesions share with the other topographies. A visible PS, a lower position of the hypothalamus, and a free chiasmatic cistern typically characterize the $3 \mathrm{~V}$-Strict group. In contrast, the $3 \mathrm{~V}$-Not-Strict category is defined by amputation or infiltration of the stalk, a position of the hypothalamus around the middle-third of the tumor, and a partial occupation of the chiasmatic cistern. Lack of visualization of the pituitary stalk was a sign common to the remaining 3 categories. However, the hypothalamus is typically observed at the upper third of the tumor in the sellar-SS and Pseudo-3V groups, whereas it is around the middle third in the Secondary-3V category. The variance of CP topography explained only by these 3 radiologic variables was $76.3 \%$.

Our scheme has proved useful for an accurate characterization of the CP-TVF relationships, which can be properly observed on the midsagittal and transinfundibular-coronal MR imaging sections. ${ }^{1,5}$ Other topographic classifications are based on the relative position of alternative anatomic references such as the optic chiasm, the diaphragma sellae, or the infundibulum, as observed through a particular operational view. ${ }^{10}$ In contrast, our topographic scheme is the only one incorporating the dynamics of tumor growth by considering the original development site along the vertical pituitary-hypothalamic axis, a feature strongly related to the surgical risks and patient outcome. ${ }^{1-5}$

\section{MR Imaging Features Essential to Define CP Topography}

The multivariate model generated in this study identified $7 \mathrm{MR}$ imaging signs whose collective assessment allows an accurate prediction of CP topography in roughly $85 \%$ of cases:

\section{1) CP Occupation of the Third Ventricle}

Third ventricle involvement is a common feature of CPs, observed in 179 cases of this cohort. In each of the remaining 21 cases in which the $3 \mathrm{~V}$ was tumor-free, the $\mathrm{CP}$ extended exclusively within the sellar and/or suprasellar compartments. Hence, we propose that $3 \mathrm{~V}$ occupation should be the first radiologic variable scrutinized on preoperative MR images to immediately identify a sellar-suprasellar location.

\section{2) Type of Pituitary Stalk Distortion}

The appearance of the PS on preoperative MR images is a fundamental variable to accurately predict $\mathrm{CP}$ topography. A not-visible PS suggests any of the 3 topographic categories that originate beneath the TVF (sellar-suprasellar, pseudointraventricular, and secondary intraventricular varieties), whereas a normal PS appearance points to the strictly intraventricular category. Alternatively, a PS whose upper infundibular edge has been amputated by the tumor or a PS wholly visible but infiltrated by the tumor represent 2 patterns strongly associated with the not-strictly intraventricular (infundibulo-tuberal) category, which is the most common topography.

\section{3) Level of the Hypothalamus Relative to the Tumor}

The position of the hypothalamus in relation to the tumor is best defined on transinfundibular-coronal scans. The position of this structure around the lower third of a CP strongly indicates a strictly intraventricular location, whereas a position of 


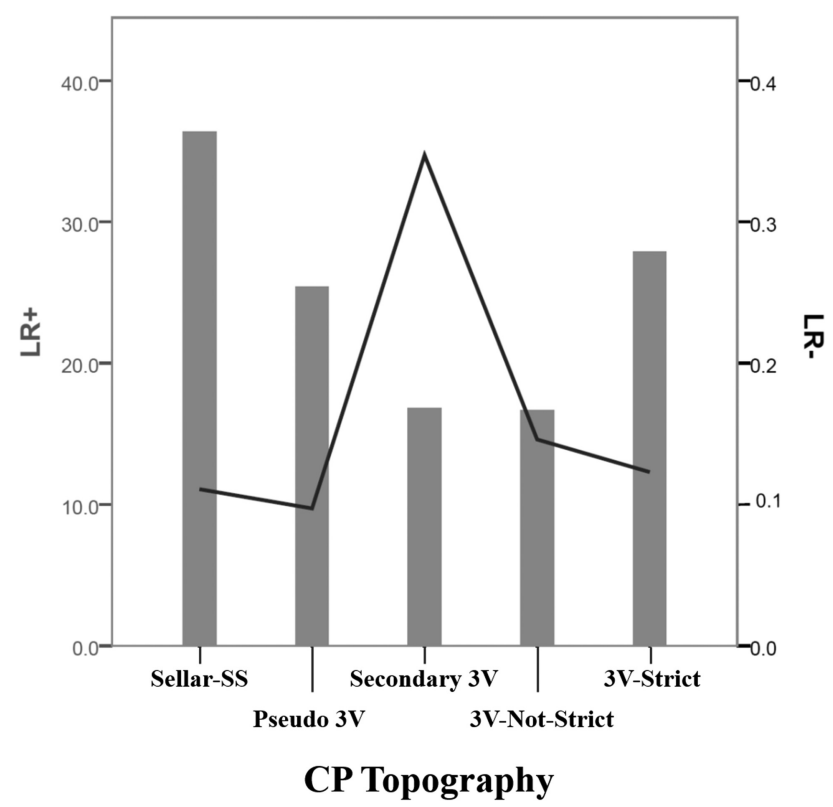

FIG 4. Diagnostic accuracy of CP topography quantified with likelihood ratios after using the 7 radiologic variables showing the strongest association (variables included in the Table). The higher the positive likelihood ratio ( $\mathrm{LR}+$, gray bars), the more reliable the topographic diagnosis is for the category considered. The lower the negative likelihood ratio ( $\mathrm{LR}-$, black line), the more reliable it is to rule out the category considered. Note that all topographic categories were associated with $L R+$ values higher than 10, though the highest figures were observed for the sellar-suprasellar (Sellar-SS), pseudointraventricular (Pseudo-3V), and strictly intraventricular (3V-Strict) categories. LR - was lower than 0.2 for all topographic categories except the secondary intraventricular one (Secondary-3V). Thus, the highest accuracy of the multivariate model of topographic diagnosis, including 7 MR imaging variables, was for the Sellar-SS, Pseudo3V, and $3 \mathrm{~V}$-Strict categories, and the lowest accuracy was for the Secondary-3V category.

the hypothalamus above the upper third of the tumor points to the topographic categories that develop below the TVF (the sellar-suprasellar and pseudointraventricular ones). In contrast, the hypothalamus is usually observed around the midthird portion or equator of the tumor, in infundibulotuberal CPs that originate within the TVF itself, or in some secondary intraventricular tumors.

\section{4) Chiasmatic Cistern Occupation}

$\mathrm{CP}$ extension into the arachnoid cistern beneath the chiasm and infundibulum is a useful variable to predict the tumor topography. The most valuable sign is a tumor-free patent cistern, which strongly indicates a strictly intraventricular position. In contrast, partial occupation of the cistern is frequently seen on the MRIs of patients with infundibulo-tuberal CPs. A cistern wholly obliterated by a tumor may be observed in any of the 3 topographic categories that originate in the sellar and/or suprasellar compartments.

\section{5) Mammillary Body Angle}

In an earlier study that included intraventricular CPs, we proved that the type of mammillary body displacement caused by these tumors was a useful sign for predicting their topography. ${ }^{6}$ The utility of this sign has been confirmed in this cohort. An obtuse angle $\left(\geq 90^{\circ}\right)$, which is related to the upward displacement of the TVF by the tumor, strongly indicates a pseudointraventricular topography. Conversely, none of the intraventricular CPs and only $10 \%$ of secondary intraventricular tumors had an obtuse deformation of the MBA. Among the intraventricular categories, hyperacute angles $\left(<30^{\circ}\right)$ generally indicate an infundibulo-tuberal (not strictly intraventricular) topography (Table).

\section{6) Type of Chiasm Distortion}

In a previous study, we defined 6 major types of chiasm distortion caused by CPs that were found to be correlated with both the pattern of visual disturbances and the tumor topography. ${ }^{7}$ The present analysis substantiates the association between the chiasm deformation and CP topography. A compressed type of chiasm distortion was typically associated with tumors originating within the TVF. Specifically, a downwardly compressed chiasm indicates the strictly intraventricular topography, whereas a chiasm compressed forward suggests the not strictly intraventricular type. Conversely, a chiasm displaced upward and showing a stretched deformation by the tumor is usually observed with CPs originating beneath the chiasm at the sellar or suprasellar compartments. Another possible pattern is a forward-stretched distortion of the chiasm, frequently caused by the secondary intraventricular topography.

\section{7) Tumor Shape}

Our multivariate model selected tumor shape as the last variable that should be evaluated on preoperative MR images to improve the diagnostic accuracy of CP topography. A round, smooth tumor shape was most frequently observed in the strictly intraventricular category. A multilobulated tumor morphology was generally found among lesions expanding within the sellar and/or suprasellar area. CPs with a pearlike outline usually originated in the sellar compartment and pushed the diaphragma sellae upward into the chiasmatic cistern.

\section{MR Imaging Accuracy to Discern CP Topography}

This study shows that the methodic assessment of 7 MR imaging variables, easily identifiable on conventional T1- and T2weighted midsagittal and coronal scans, permits a correct topographic diagnosis in $86 \%$ of cases. Nevertheless, the accuracy of our MR imaging multivariate model to preoperatively define the exact tumor location varies for each topographic category. The discriminative power of our model was dramatically increased for the sellar-suprasellar, pseudointraventricular, and strictly intraventricular categories. A correct topographic diagnosis was made in $90 \%$ of the tumors within these 3 categories. In contrast, the secondary intraventricular topography could be accurately predicted in $<70 \%$ of cases. This lower accuracy rate can be explained by the highly variable range of anatomic distortions caused by this topographic subgroup (Fig 3). A secondary intraventricular category should be presumed when a preoperative $M R$ imaging shows a CP extending from the sella turcica into the $3 \mathrm{~V}$ and the PS is not visible (occurring in $92 \%$ of these cases). A hypothalamus positioned around the midthird portion of the lesion (in 77\%) and a multilobulated shape (58\%) are other signs usually shown by this category.

With the aim of achieving an optimal preoperative prediction 
for CP topography, specific MR imaging sequences should be obtained, in addition to the conventional T1- and T2-weighted ones. The heavily T2-weighted and the fast-imaging employing steadystate acquisition MR imaging sequences represent the 2 methods that offer the finest resolution of the CP-brain contact surfaces, as well as the best definition of the distorted neurovascular structures. ${ }^{11-14}$ In particular, the high-resolution 3D-FIESTA sequence has proved invaluable to ascertain the accurate anatomic relationship between a CP and the TVF. ${ }^{13}$ The resolution power of this technique to correctly define the displacement pattern of the mammillary bodies and optic chiasm, 2 structures not visible in around 15\% of cases with routine T1- and T2-weighted MRI, should be further investigated in a large series. High-resolution 3D-FIESTA/CISS sequences could quite possibly improve the accuracy of diagnosis in large CPs presenting MR imaging features shared by several topographies. ${ }^{14}$

\section{CONCLUSIONS}

A precise description of CP topography is essential to guide a surgeon's judicious decision regarding the approach to the tumor and degree of removal suitable to avoid undue injury to the hypothalamus. Seven MR imaging variables should be analyzed to obtain an accurate preoperative definition of CP topography: third ventricle occupation by the tumor, pituitary stalk appearance, level of the hypothalamus relative to the tumor, chiasmatic cistern occupation, mammillary body angle, type of chiasm distortion, and tumor shape. The systematic assessment of these variables on midsagittal and coronal conventional T1- and T2weighted MR images allows an accurate topographic classification in $86 \%$ of cases.

\section{ACKNOWLEDGMENTS}

The authors are grateful to George Hamilton for his critical review of the language and style of the manuscript.

\section{REFERENCES}

1. Pascual JM, González-Llanos F, Barrios L, et al. Intraventricular craniopharyngiomas: topographical classification and surgical approach selection based on an extensive overview. Acta Neurochir (Wien) 2004;146:785-802 Medline

2. Pascual JM, Prieto R, Castro-Dufourny I, et al. Topographic diag- nosis of papillary craniopharyngiomas: the need for an accurate MRI-surgical correlation. AJNR Am J Neuroradiol 2015;36: E55-56 CrossRef Medline

3. Pascual JM, Carrasco R, Prieto R, et al. Craniopharyngioma classification. J Neurosurg 2008;109:1180-82; author reply 1182-83 CrossRef Medline

4. Pascual JM, Prieto R, Carrasco R. Infundibulo-tuberal or not strictly intraventricular craniopharyngioma: evidence for a major topographical category. Acta Neurochir (Wien) 2011;153:2403-25; discussion 2426 CrossRef Medline

5. Prieto R, Pascual JM, Rosdolsky M, et al. Craniopharyngioma adherence: a comprehensive topographical categorization and outcome-related risk stratification model based on the methodical examination of $\mathbf{5 0 0}$ tumors. Neurosurg Focus 2016;41:E13 CrossRef Medline

6. Pascual JM, Prieto R, Carrasco R, et al. Displacement of mammillary bodies by craniopharyngiomas involving the third ventricle: surgical-MRI correlation and use in topographical diagnosis. J Neurosurg 2013;119:381-405 CrossRef Medline

7. Prieto R, Pascual JM, Barrios L. Optic chiasm distortions caused by craniopharyngiomas: clinical and magnetic resonance imaging correlation and influence on visual outcome. World Neurosurg 2015; 83:500-29 CrossRef Medline

8. Prieto R, Pascual JM, Castro-Dufourny I, et al. Craniopharyngioma: surgical outcome as related to the degree of hypothalamic involvement. World Neurosurg 2017;104:1006-10 CrossRef Medline

9. Pascual JM, Prieto R, Castro-Dufourny I, et al. Development of intracranial approaches for craniopharyngiomas: an analysis of the first 160 historical procedures. Neurosurg Focus 2014;36:E13 CrossRef Medline

10. Lubuulwa J, Lei T. Pathological and topographical classification of craniopharyngiomas: a literature review. J Neurol Surg Rep 2016;77: e121-127 CrossRef Medline

11. Saeki N, Murai H, Kubota M, et al. Heavily T2 weighted MR images of anterior optic pathways in patients with sellar and parasellar tumors: prediction of surgical anatomy. Acta Neurochir (Wien) 2002;144:25-35 CrossRef Medline

12. Watanabe K, Kakeda S, Yamamoto J, et al. Delineation of optic nerves and chiasm in close proximity to large suprasellar tumors with contrast-enhanced FIESTA MR imaging. Radiology 2012;264: 852-58 CrossRef Medline

13. Xie $\mathrm{T}$, Zhang XB, Yun $\mathrm{H}$, et al. 3D-FIESTA MR images are useful in the evaluation of endoscopic expanded endonasal approach for midline skull-base lesions. Acta Neurochir (Wien) 2011;153:12-18 CrossRef Medline

14. Gu Y, Zhang X. Mamillary body angle and craniopharyngioma. J Neurosurg 2014;120:1241-43; author reply 1243-45 CrossRef Medline 